\title{
Mild COVID-19 and Impaired Blood Cell-Endothelial Crosstalk: Considering Long-Term Use of Antithrombotics?
}

\author{
Arthur Melkumyants ${ }^{1,2}$ Ludmila Buryachkovskaya ${ }^{1}$ \\ Victor Serebruany ${ }^{4}$ \\ ${ }^{1}$ Cell Adhesion Department, National Medical Research Center of \\ Cardiology, Moscow, Russia \\ 2 Department of Physics of Living Systems, Institute of Physics and \\ Technology, Moscow, Russia \\ ${ }^{3}$ Cardiology Division, Central Clinical Hospital of Presidential \\ Administration, Moscow, Russia \\ ${ }^{4}$ Division of Neurology, Johns Hopkins University School of Medicine, \\ Baltimore, Maryland, United States
}

\author{
Nikita Lomakin ${ }^{3}$ Olga Antonova ${ }^{1}$
}

Thromb Haemost 2022;122:123-130.
Abstract
Keywords
- COVID-19
- circulating endothelial cells
- erythrocytes
- echinocytes
- electron microscopy
- flow cytometry

\author{
Address for correspondence Victor Serebruany, MD, PhD, Division of \\ Neurology, Johns Hopkins University School of Medicine, 14110 Rover \\ Mill Road, West Friendship, MD 21794, United States \\ (e-mail: vserebr1@jhmi.edu; heartdrug@aol.com).
}

received

April 28, 2021

accepted after revision

July 13,2021

published online

July 16, 2021
Background Current coronavirus disease 2019 (COVID-19) pandemic reveals thrombotic, vascular, and endothelial dysfunctions at peak disease. However, the duration, degree of damage, and appropriate long-term use of antithrombotic strategies are unclear. Most COVID data are yielded from random clinical observations or autopsy of postmortem samples, while precise blood cellular data in survivors are insufficient.

Methods We analyzed erythrocytes, circulating endothelial cells, and echinocytes by electron microscopy and flow cytometry in patients with confirmed COVID-19 $(n=31)$ and matched healthy controls $(n=32)$ on admission and at hospital discharge.

Results All patients experienced mild disease, none required pulmonary support, and all survived. Admission number of circulating endothelial cells was significantly (40100 times) higher in COVID-19 patients. Cells were massively damaged by multiple fenestrae in membranes with diameter comparable to the size of supercapsid in SARSCoV-2 (severe acute respiratory syndrome coronavirus 2) virus. COVID-19 also provoked formation of stacked aggregated erythrocytes capable of clogging microvascular bed and of diminishing oxygen supply. In some patients, such abnormalities persisted at hospital discharge revealing remaining intracellular penetration of SARS-CoV- 2 where it may be replicated and returned to circulation.

Conclusion These observational and descriptive data suggest that persistent viral cell injury may cause blood vessel damage; their increased permeability resulted in tissue edema, inflammation, platelet activation, and augmented thrombosis. There is a residual blood cell damage following the acute phase in some COVID-19 survivors. Controlled outcome-driven trials are urgently needed for exploring optimal use of longterm antithrombotics and vascular protection strategies even after mild COVID-19. 


\section{Introduction}

At the end of 2019, a novel coronavirus infection COVID-19 (coronavirus disease 2019) broke out in China's central province of Hubei. The pandemic was heralded by World Health Organization on March 11, 2020. A single-stranded RNA virus, SARS-CoV-2 (severe acute respiratory syndrome coronavirus 2), which causes this disease evokes a severe pneumonia that is characterized by a high-grade fever and bad dry cough in 15 to $20 \%$ cases. Such COVID-19 pneumonia frequently causes acute respiratory distress syndrome ${ }^{1}$ and/or multiorgan failure ${ }^{2}$ associated with high mortality risk. However, pneumonia is not a unique disease provoked by COVID-19. Accumulated clinical data and some autopsy evidence suggest that this infection also affects the cardiovascular system, inducing acute myocardial infarctions, myocarditis, pericarditis, and severe arrhythmias. ${ }^{3}$ Lately, COVID-19 has been under investigation for causing disturbances in cell-endothelial crosstalk. ${ }^{4,5}$ The systemic damage is spread far beyond the heart, impacting the kidneys, ${ }^{6}$ liver, ${ }^{7}$ pancreas, ${ }^{8}$ and severe neurological disturbances, ${ }^{9,10}$ clearly suggesting multiorgan impairment.

Over time the clinical experience and autopsy data clearly established that the critical cases of COVID-19 are characterized by pronounced coagulopathy, hypoxia, and endothelial dysfunction. ${ }^{11,12}$ In fact, COVID-19 is currently described not only as a pulmonary disease, but also as coagulopathy and vasculitis, or more specifically, as endothelial damage. ${ }^{1,11,13}$ However, the validity of such assumption was evidenced exclusively by random (not randomized) clinical observations, conventional blood tests, and sporadic autopsy data. We utilized scanning electron microscopy in combination with flow cytometry to assess interactions between circulating blood cells (erythrocytes, circulating endothelial cells [CECs], and echinocytes) in hospitalized COVID-19 patients at hospital admission and discharge.

\section{Methods}

\section{Patients}

All study participants provided the informed consent, which was performed in strict adherence to ethical directives and regulations of World Medical Association Declaration of Helsinki (2013) and according to the Directive No. 266 (June 19, 2003) of Ministry of Health of the Russian Federation "On Establishing the Rules of Clinical Practice in the Russian Federation." All research protocols were approved by Central Clinical Hospital of Presidential Administration Ethical Committee. Thirty-one patients with polymerase chain reaction-confirmed COVID-19 diagnosis were admitted to the hospital from May 25 to July 22, 2020 and included in a single-center prospective observational study. Thirty-two COVID-19-negative samples from matched demographic personnel constituted the control group. During the hospital stay all patients survived, and no patient was referred to the intensive care unit (ICU) or required pulmonary ventilation. All patients were discharged from the hospital on average of approximately 2 weeks frame (range 10-16 days). Two specimens (admission and discharge) of venous blood were collected for subsequent electron microscopy and flow cytometry testing.

\section{Electron Microscopy}

Blood was drawn from the cubital vein into the VACUETTE tubes (Greiner bio-one, Austria) containing sodium citrate (3.2\%). Immediately after this, sample fixation was performed with glutaraldehyde $(0.1 \%, 4.5 \mathrm{~mL}$, Sigma). After sedimentation of some RBCs for 20 minutes at room temperature, $20 \mu \mathrm{L}$ was collected from the upper layer and placed into $2.5 \%$ glutaraldehyde for complete fixation prior to performing scanning electron microscopy. Polycarbonate isopore membrane filters of 0.22 to $0.45 \mu \mathrm{m}$ diameter were utilized as the matrices for $\mathrm{RBC}$. Then a quarter of the filter was cut out to count the total number of CECs. To control precision of CEC scoring, the cells were recounted by flow cytometry. Specifically, CECs were scored with the method of sequential gaiting, which we modified to count not only intact, but also the damaged endothelial cells (ECs). The cells were examined with a scanning electron microscope (Inspect F50; FEI Company, Eindhoven, The Netherlands) and X-Max EDS-Detector (Oxford Instruments, Abingdon, United Kingdom). Since the scatter in this score of desquamated EC was rather large due to the small volume of the specimens, we assessed the accuracy of the electron microscopy data with flow cytometry.

\section{Flow Cytometry}

Blood was collected in $3.8 \%$ sodium citrate at a blood/anticoagulant ratio of 9:1. Samples of whole blood (50 $\mu \mathrm{L}$ ) were incubated with antibodies CD45-FITC as a pan-leukocyte marker, CD42b-APC (GPIb) as a platelet marker, and CD31PE (all Becton Dickinson, BD Biosciences, San Jose, California, United States) as an EC marker at concentrations recommended by the manufacturer for 30 minutes at room temperature in the dark. Erythrocytes were lysed by adding to the samples $900 \mu \mathrm{L}$ of cooled $\left(4^{\circ} \mathrm{C}\right)$ distilled water. After pipetting for 20 seconds, isotonicity of the cell suspension was restored by adding $300 \mu \mathrm{L}$ of $\mathrm{NaCl}$ saline $(0.6 \mathrm{M})$, and the volume was adjusted up to $2 \mathrm{~mL}$ with phosphate-buffered saline (PBS) $(150 \mathrm{mM} \mathrm{NaCl}$, $10 \mathrm{mM}$ sodium phosphate, $\mathrm{pH}$ 7.4). Then the cells were sedimented by centrifuging at $300 \mathrm{~g}$ for 5 minutes at room temperature and resuspended for assay in a final volume of $300 \mu \mathrm{L}$ in paraformaldehyde solution (1\%) mixed with $2 \%$ bovine serum albumin/PBS. Immediately prior to analysis, the specimen was supplemented with $50 \mu \mathrm{L}$ suspension of 10 - $\mu \mathrm{m}$ reference particles (Flow-Count Fluorospheres, Beckman Coulter, Brea, California, United States). The concentration of the particles in this suspension was used to scale the cell scores. CECs were scored using a FACS Canto II flow cytometer and BD CELLQuest (both Becton Dickinson, BD Biosciences) software. The CEC score was determined with a sequential gating method and defined as $\mathrm{CD} 45^{-} \mathrm{CD} 31^{+} \mathrm{CD}_{2} \mathrm{~b}^{-}$. Analysis was performed until 1,000 events were counted in the gate of reference particles and the results are presented in - Fig. 4. Due to the presence of limited number of CD31 antigens on the platelet membrane, CECs were determined as a fraction with negative platelet marker CD42b (dot plot CD42b-APC/CD31-PE, gate Q4-1) and analyzed as 


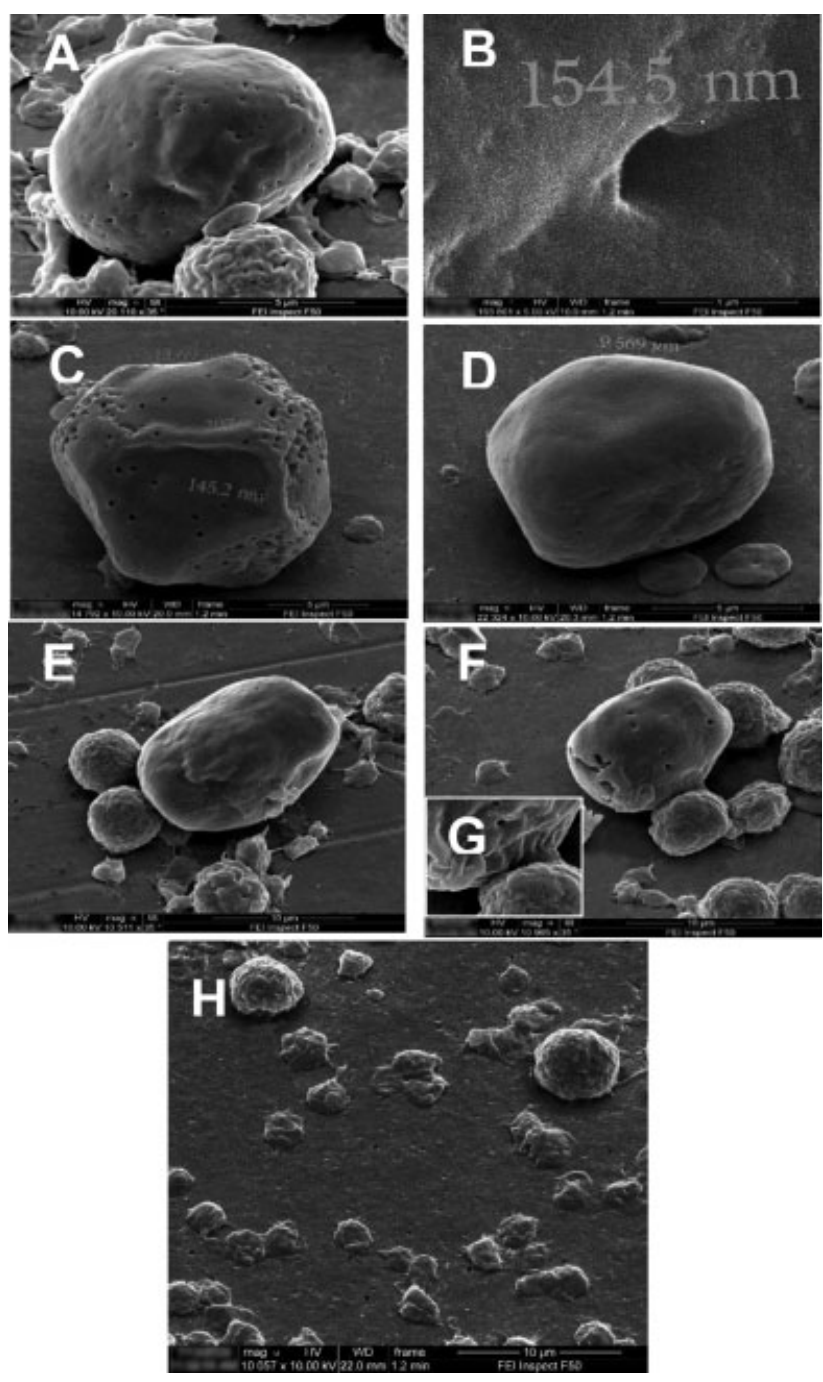

Fig. 1 Circulating endothelial cells in COVID-19 patients. Circulating endothelial cell with typical fenestrated membrane $(\mathbf{A})$ and the same cell at a greater magnification (B) exhibiting an orifice in the membrane corresponding to the size of supercapsid of SARS-CoV-2.

Circulated endothelial cells of two clinically similar patients are shown in panels $C, D$ and in E, F. Pictures are made on admission (C, E) and discharge $(D, G)$. Control cells are shown in $(H)$. Magnification of all pictures at $\times 10,000$.

$\mathrm{CD} 45^{-} \mathrm{CD} 31^{+} \mathrm{CD} 42 \mathrm{~b}^{-}$fraction (C2, C3). Since COVID-19 provokes a pronounced endothelial dysfunction and platelet aggregation, this sequential gating was employed to active adhered platelets and their aggregates in the gate of mononuclear cells. Finally, CD45-negative fraction Q4 was selected in gate P2 on CD45-FITC/CD31-PE dot plot to further score as $\mathrm{CD} 45^{-} \mathrm{CD} 31^{+} \mathrm{CD} 42 \mathrm{~b}^{+}$fraction in gate Q2-1 $(\mathrm{C} 2, \mathrm{C} 3)$. The platelet fraction was detected in all patients (C3) but not reported here.

\section{Statistics}

Continuous variables were described as mean ( \pm standard error) and categorical variables as percentages. Categorical variables were compared by the Chi-square test. All variables before and after the end of therapy were compared applying the nonparametric Mann-Whitney test. All tests were twotailed and $p<0.05$ was considered statistically significant.
Statistical analyses were performed using IBM SPSS Statistics for Windows, Version 24.0. (Chicago, Illinois, United States)

\section{Results}

The admission variables of demographics and comorbidities in the COVID-19 patients and healthy controls are summarized in - Table 1. The arms match well with regard to age, gender, weight, and smoking. However, the COVID-19 patients were not only slightly older, but exhibited more hypertension, coronary artery disease, and cancer. The differences in admission and discharge laboratory indices compared with controls are summarized in - Table 2. Notably, $\mathrm{SpO}_{2}$ was always high, but both C-reactive protein (CRP) and $\mathrm{D}$-dimer were markedly elevated on admission. At discharge, CRP and D-dimer were normal.

\section{Circulating Endothelial Cells}

The electron images of CEC found in the blood of COVID-19 patients before and after the treatment are exhibited in -Fig. 1. The exhibited patient was admitted on day 9 after appearance of COVID-19 symptoms with severely damaged CEC (C). At discharge after a 10-day stay in the hospital, this patient demonstrated only solitary lesions in the membrane (D). Another patient was admitted the next day after COVID19 symptoms' manifestation with no lesions in the CEC membrane (E). Surprisingly, at discharge after a 19-day hospital stay, this patient had damaged CEC (F). The insert in panel $(G)$ demonstrates phagocytosis of CEC by neutrophils. The next image $(\mathrm{H})$ shows a typical picture of blood cells in individuals of the control group without COVID-19, when CECs were absent. Noteworthy, there are numerous holes in the CEC membrane (-Fig. 1A, C, F). Importantly, these holes were present not only on admission to the hospital but also at discharge ( - Fig. 1D, F).

During COVID-19 there was a remarkable increase of CEC numbers. In our study, the electron microscopy detected 0 to $8 \mathrm{CECs}$ in $5-\mu \mathrm{L}$ blood specimens drawn from the patients on admission, which corresponded to total CEC score of $400 \pm 40$ cells $/ \mathrm{mL}$.

The scores of CEC were $4.7 \pm 1.9$ and $380 \pm 20$ cell $/ \mathrm{mL}$, correspondingly $(p<0.0001)$ in matched controls. Both scoring methods revealed essential lesions to endothelium of COVID-19 patients indicating a risk of vascular denudation. Indeed, the CEC scores obtained with both methods differed insignificantly ( $404.6 \pm 33.8$ vs. $376.43 \pm 31.3 ; p>0.1$ ).

CEC scoring data obtained on admission and discharge from the hospital clearly differentiated the patients into two groups. In some patients $(n=24$, group 1$)$, the CEC score decreased by more than two times after the therapy from $370 \pm 20$ to $145 \pm 12$ cells $/ \mathrm{mL}(p<0.001)$. However, in some patients ( $n=7$, group 2 ) the CEC score significantly increased from $310 \pm 20$ to $390 \pm 20$ cell $/ \mathrm{mL}(p<0.05)$. While the larger $(n=24)$ portion of patients exhibited a significant $(p<0.001)$ reduction of CEC concentrations, however, a smaller subset $(n=7)$ demonstrated also a significant $(p<0.05)$ but increase in CEC numbers at discharge. In contrast, per cent of echinocytes was uniformly and significantly $(p<0.05)$ lower 
Table 1 Demographics and clinical characteristics in COVID-19 patients and controls

\begin{tabular}{|l|l|l|l|}
\hline Variable & $\begin{array}{l}\text { Controls } \\
(\boldsymbol{n}=32)\end{array}$ & $\begin{array}{l}\text { COVID-19 patients } \\
(\boldsymbol{n}=31)\end{array}$ & $\begin{array}{l}p \text {-Value for factor homogeneity } \\
\text { across samples }\end{array}$ \\
\hline Gender male/female, $n(\%)$ & $17(53 \%) / 15(47 \%)$ & $19(61 \%) / 12(39 \%)$ & 0.51 \\
\hline Age $(\mathrm{y})$ & $54.2(25-74)$ & $59.3(27-83)$ & 0.63 \\
\hline BMI $\left(\mathrm{kg} / \mathrm{m}^{2}\right)$ & $25.1(21.8-31.4)$ & $29.4(19.2-42.0)$ & 0.57 \\
\hline Obesity & $5(15.9 \%)$ & $6(19.2 \%)$ & 0.70 \\
\hline Smoking & $4(12.5 \%)$ & $1(3.2 \%)$ & 0.17 \\
\hline Hypertension & $4(12.5 \%)$ & $14(45.2 \%)$ & 0.02 \\
\hline Diabetes & - & $2(6.5 \%)$ & 0.14 \\
\hline Coronary artery disease & - & $4(12.9 \%)$ & 0.04 \\
\hline Heart failure & - & $2(6.5 \%)$ & 0.14 \\
\hline Cancer & - & $7(22.6 \%)$ & $<0.01$ \\
\hline Chronic kidney disease & - & $1(3.2 \%)$ & 0.31 \\
\hline COPD & - & $2(6.5 \%)$ & 0.14 \\
\hline
\end{tabular}

Abbreviation: COPD, chronic obstructive pulmonary disease.

Table 2 Basic biochemical and physiological parameters prior to and after the therapy of COVID-19 patients

\begin{tabular}{|c|c|c|c|c|}
\hline \multirow[t]{2}{*}{ Variable } & \multirow[t]{2}{*}{ Controls $(n=32)$} & \multicolumn{3}{|c|}{ COVID-19 patients $(n=31)$} \\
\hline & & Admission & Discharge & $p$-Value \\
\hline Heart rate $\left(\min ^{-1}\right)$ & $74.3 \pm 2.1$ & $85.2 \pm 2.7$ & $76.2 \pm 1.9$ & $<0.01$ \\
\hline Respiratory rate $\left(\mathrm{min}^{-1}\right)$ & $16.5 \pm 0.8$ & $17.8 \pm 0.9$ & $17.0 \pm 0.7$ & 0.42 \\
\hline Temperature (C) & $36.2 \pm 0.3$ & $37.6 \pm 0.3$ & $36.5 \pm 0.4$ & $<0.01$ \\
\hline $\mathrm{SpO}_{2}(\%)$ & $97.0 \pm 1.0$ & $94.4 \pm 0.8$ & $96.2 \pm 0.5$ & 0.71 \\
\hline Hemoglobin $(\mathrm{g} / \mathrm{L})$ & $127.8 \pm 4.5$ & $135.2 \pm 3.9$ & $128.4 \pm 2.7$ & 0.28 \\
\hline Leukocytes $\left(10^{9} / \mathrm{L}\right)$ & $4.7 \pm 0.6$ & $6.1 \pm 1.9$ & $6.1 \pm 1.6$ & 0.09 \\
\hline Platelets $\left(10^{9} / \mathrm{L}\right)$ & $192.0 \pm 7,5$ & $189.5 \pm 9.6$ & $236.1 \pm 10.8$ & $<0.01$ \\
\hline Lymphocytes (\%) & $28.4 \pm 2,9$ & $23.6 \pm 3.1$ & $30.7 \pm 3.7$ & 0.05 \\
\hline Neutrophils (\%) & $54.4 \pm 1.9$ & $66.4 \pm 4.7$ & $57.6 \pm 5.2$ & 0.17 \\
\hline $\mathrm{ESR}(\mathrm{mm} / \mathrm{h})$ & $12.0 \pm 3.1$ & $28.2 \pm 1.9$ & $23.5 \pm 1.7$ & 0.02 \\
\hline CRP (mg/L) & $4.2 \pm 0.6$ & $21.1 \pm 4.2$ & $2.2 \pm 1.1$ & $<0.01$ \\
\hline Creatinine $(\mathrm{mg} / \mathrm{dL})$ & $82.5 \pm 4.3$ & $91.9 \pm 2.9$ & $90.0 \pm 2.6$ & 0.08 \\
\hline $\mathrm{pH}$ & $7.41 \pm 0.03$ & $7.34 \pm 0.02$ & $7.38 \pm 0.02$ & 0.27 \\
\hline Lactate (mmol/L) & $1.8 \pm 0.3$ & $2.7 \pm 0.5$ & $3.1 \pm 0.4$ & 0.32 \\
\hline D-dimer (ng/L) & $328.0 \pm 15.2$ & $472.1 \pm 14.3$ & $231.3 \pm 10.7$ & $<0.01$ \\
\hline Fibrinogen $(\mathrm{g} / \mathrm{L})$ & $2.9 \pm 0.3$ & $4.7 \pm 0.7$ & $3.8 \pm 0.4$ & 0.04 \\
\hline Ferritin $(\mu \mathrm{g} / \mathrm{L})$ & $85.4 \pm 8.9$ & $247.4 \pm 18.2$ & $363.1 \pm 22.3$ & $<0.01$ \\
\hline
\end{tabular}

Abbreviations: CRP, C-reactive protein; ESR, erythrocyte sedimentation rate; $\mathrm{SpO}_{2}$, peripheral blood oxygen saturation.

after hospitalization. No correlation has been established between the conventional inflammation biomarkers and CEC numbers at discharge.

\section{Erythrocytes}

Changes in RBC observed in patients with COVID-19 were most commonly manifested by the formation of long lengthy stacked aggregated cells (erythrocyte sludge) and by the transformation of shape of part of RBC from normal biconcave discocytes to echinocytes.

-Fig. 2 shows stacked RBC (blood sludge) formation, which was typical for all examined patients. The number of cells in RBC rouleaux in patients examined on admission to the hospital ranged from 3 to 12 cells. On discharge, the RBC stacks consisted of maximum 3 cells in 24 patients, while it ranged from 4 to 5 in other 7 patients. It is noteworthy that 


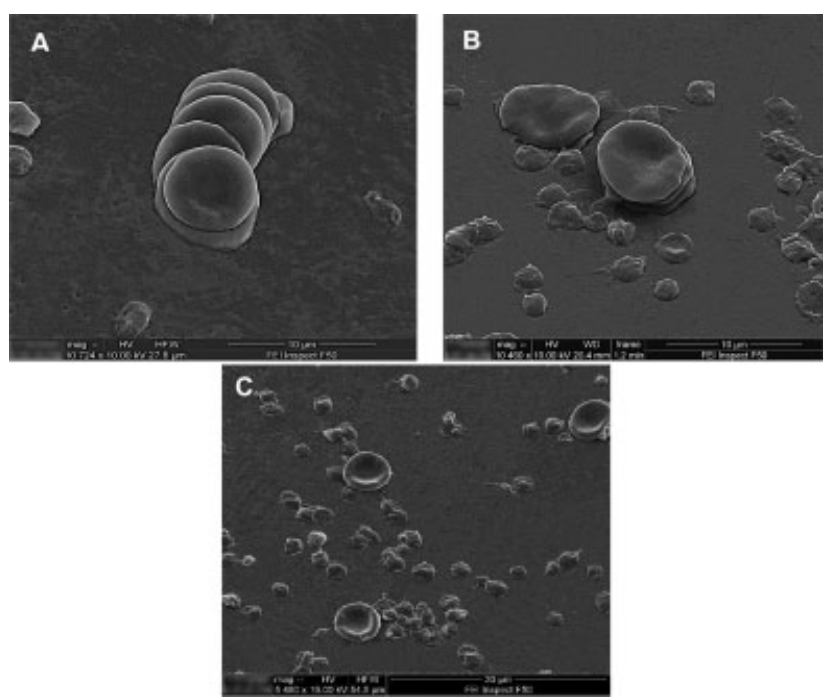

Fig. 2 Stacked red blood cells (RBCs) on admission and discharge from the hospital. (A) Stacked red blood cells with 7 erythrocytes in the blood specimen obtained on admission and (B) stacked cells with 3 erythrocytes in the blood specimen of the same patient at discharge. Red blood cells here have the discocyte form. Magnification: $\times 10,000$. (C) Typical picture of blood cells in controls, stacked RBCs are absent. Magnification: $\times 5,000$.
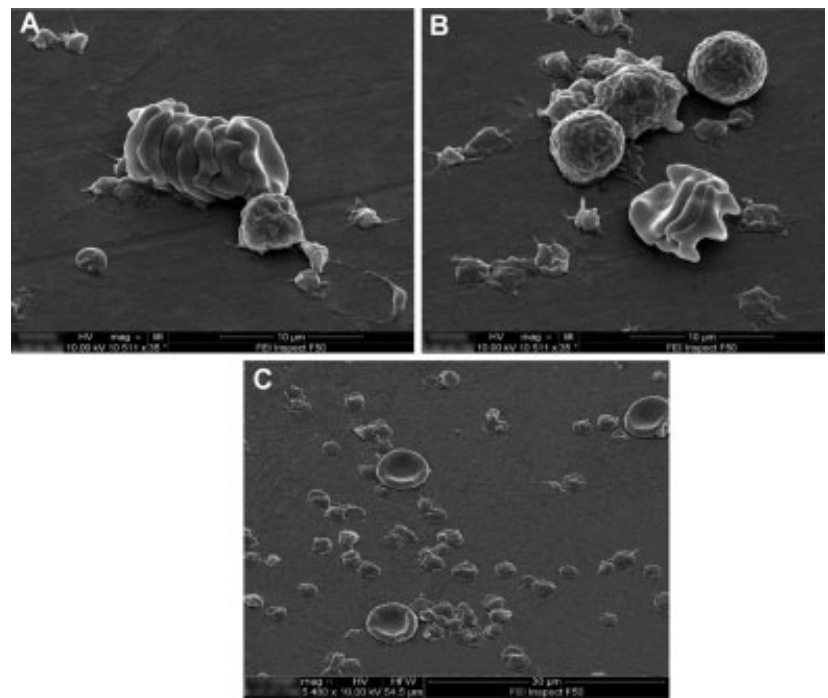

Fig. 3 Red blood cells transformed into echinocytes. (A) Stacked red blood cells with 7 echinocytes in the blood specimen obtained on admission and (B) stacked red blood cells with 3 echinocytes in the blood specimen of the same patient at discharge. Magnification: $\times 10,000$. (C) Typical picture of blood cells in individuals from the control group, echinocytes are absent. Magnification: $\times 5,000$.

the "long" RBC rouleaux remained only in patients ( $n=17$ or $56 \%$ ) with blood $\mathrm{pH}$ ranging from 7.28 to 7.34 , which was below the level of 7.35. In contrast, the "short" RBC rouleaux remained in patients ( $n=14$ or $44 \%$ ) with blood $\mathrm{pH}$ ranging from 7.36 to 7.54 . The RBC score was $4.41 \pm 0.4 \times 10^{9}$ in COVID -19 patients and $4.28 \pm 0.56 \times 10^{9}$ in controls. These differences were not significant $(p>0.1)$. The number and length of sludges also did not correlate with such an inflammatory biomarker as CRP.

\section{Echinocytes}

Most patients ( $n=25$ or $81 \%$ ) demonstrated a pronounced transformation of some erythrocytes into echinocytes both on admission and discharge from the hospital. On admission, the share of echinocytes was $18 \pm 4 \%$ total RBC score. At discharge, this share significantly decreased to $12 \pm 2 \%$ $(p<0.05)$ albeit remaining rather high ( - Fig. 3 , right panel).

\section{Discussion}

There are few important advances raised by the index study. First, the definite proof of impaired vascular endotheliumcell crosstalk in COVID-19 patients has been documented not postmortem nor by routine clinical examination, but directly by electron microscopy confirmed by cell flow cytometry in survived mild-to-moderately sick hospitalized patients. Indeed, most COVID-19 electron microscopy studies were conducted in autopsy samples ${ }^{1,2}$ rather than by directly examining life cells. Second, the alarming signal that COVID-19 still impacts blood cell structure after hospital discharge may be critical to explain delayed complications such as thrombosis, sudden deaths, etc. Third, such substantial cell disturbances existed even in mild COVID-19. In fact, none of our patients died, or required life support. Most likely terminal patients on lung ventilation will exhibit even more damage, but the degree of dysfunction in the mild cohort utilized in our study is truly unexpected and alarming.

The hypothesis that blood cell dysfunction may be a cornerstone in the progression of SARS-CoV-2 virus infection and further vascular damage is reasonable, since COVID-19 predecessor (SARS-CoV virus) binds in the lung to the angiotensin-converting enzyme 2 (ACE-2) receptor. ${ }^{14,15} \mathrm{By}$ perforating the alveolar-capillary membrane, the virus enters the vascular bed and then, moving with the flow of blood, can reach other organs, on the vascular endothelium of which the ACE-2 receptor is sufficiently represented. The diameter of these orifices $(100-200 \mathrm{~nm})$ roughly corresponded to that of SARS-CoV2 supercapsid. ${ }^{16}$ This fact suggests that the holes may be produced by a virus penetrated into the endotheliocyte, repeatedly replicated in it, damaging the cell and then leaving it. Importantly, ACE-2 receptors are present on the lung alveolar epithelial cells, and in epithelial cells of small intestine; vascular EC and smooth muscle cells of all organs; basal cutaneous epidermis; and nasal and oral mucous coats. ${ }^{17}$ The last association probably explains classical loss of smell and taste following COVID-19 infection.

Some earlier hypotheses suggested that the "viral stage" of the disease targets lungs almost exclusively, while the nature of COVID-19 general vascular multiorgan component is secondary. The index data challenge such hypothesis since virtually all CECs exhibited numerous fenestrae on the membrane (-Fig. 2) with the diameter matching those of SARS-CoV-2 supercapsids. ${ }^{16,18}$

An elevated CEC score during viral infections and especially in septic shock is already described. ${ }^{19}$ In fact, this index rose pronouncedly from 1 to 15 cells $/ \mathrm{mL}$ in healthy persons to an average of 40 to 50 cells/mL in hospitalized COVID-19 patients. In this regard, two important facts should be noted. 


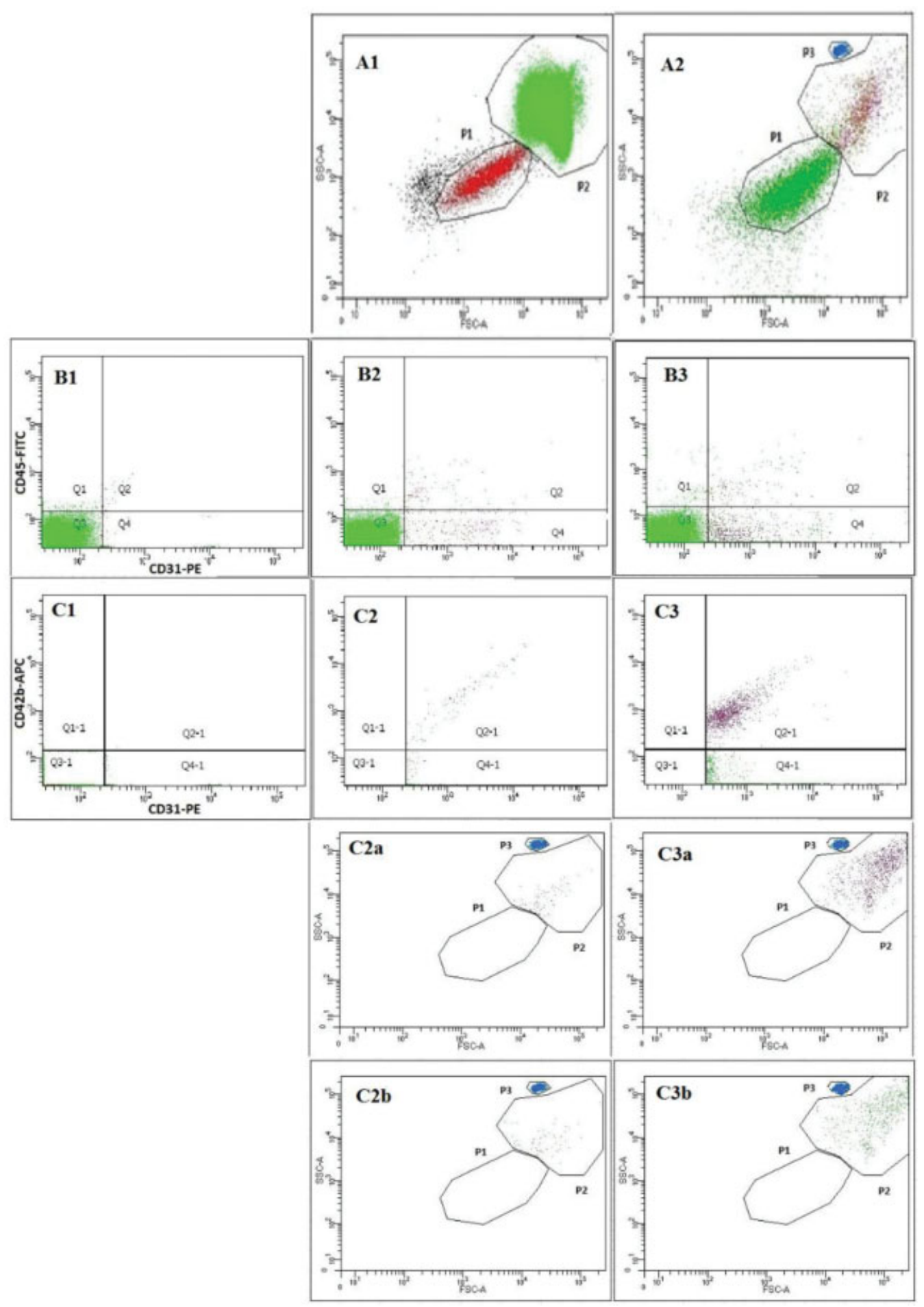

Fig. 4 Flow cytometry of circulating endothelial cells: sequential gating and analysis of cell fractions. A1: whole blood; $A 2$ : probes after erythrocyte lysis; B1, C1: negative controls; B2, C2: healthy donors; B3, C3: patients with COVID-19. In SSC/FSC gate scattering: platelets (P1), mononuclear cells (P2), reference particles (P3). Circulating endothelial cells are scored in gate P2 with a CD45-FITC/CD31-PE dot plot as CD45negative fraction Q4. Since there is limited number of CD31 antigens on the platelet membrane, circulating endothelial cells are measured as a fraction with negative platelet marker CD42b (dot plot CD42b-APC/CD31-PE, gate Q4-1) and analyzed as $C D 45^{-} \mathrm{CD} 31^{+} \mathrm{CD} 42 \mathrm{~b}^{-}$fraction (C2, C3, $\mathrm{C} 2 \mathrm{~b}, \mathrm{C} 3 \mathrm{~b}$ ). The sequential gating was utilized to assess CD45-negative fraction Q4 selected in gate P2 on the CD45-FITC/CD31-PE dot plot to further score as $\mathrm{CD} 45^{-} \mathrm{CD} 31^{+} \mathrm{CD} 42 \mathrm{~b}^{+}$fraction in gate Q2-1 (C2, C3, C2a, C3a). The platelet fraction was detected in all patients (C3, C3a). The gates for platelets, mononuclear cells, and reference particles are marked as P1, P2, and P3.

First, the mean score of CEC was significantly higher in patients referred to ICUs. ${ }^{20}$ Second, the individual CEC scores were characterized by a pronounced data scattering. In addition, the non-ICU patients were divided into two groups: the major one had a very low average score of CEC $(<20$ cells $/ \mathrm{mL}$ ), while in the minor group this score amounted to 300 to 400 cells/mL. Paradoxically, such high scores were not typical of ICU patients. ${ }^{21}$ 
Our data essentially differ from the abovementioned evidence. Here, the mean CEC score of 300 to 400 cells $/ \mathrm{mL}$ corresponds to maximal values reported in other papers. ${ }^{22,23}$ It is noteworthy that the patients examined in our study were not critically sick. Probably, the CEC score inconsistencies were caused by specific differences in scoring methods employed by other studies. Actually, virtually all evidence focused on analysis of CEC score in various pathologies that employed the methods considering CEC as mature, healthy, functionally adequate, nucleated, and antigen-expressing cells. ${ }^{24}$ Evidently, CECs detected with the electron microscopy in the present study look like the damaged cell skeletons, which could be omitted in scoring performed with routine flow cytometry. ${ }^{24}$ This was the reason why we scored CEC with sequential identification of flow cytometry data utilizing sequential gating.

The CEC scores calculated by electron microscopy and modified flow cytometry attest to coherence and adequacy of both approaches. Our data strongly suggest that vascular traumas caused by SARS-CoV-2 result in pronounced cell dysfunction and potential endothelial denudation leading to intensive intravascular thrombosis and alteration of RBC accompanied by disturbances in regional gas exchange culminating in pronounced ischemia.

Severe damage to circulatory and hematological indices inflicted by COVID-19 is most strikingly manifested by a large number of circulating RBC rouleaux (see - Fig. 4 for details). Indeed, this pathological pattern is typical of the patients infected with novel coronavirus. Two other important features should be briefly mentioned. First, aggregation of RBCs is reversible, so removal of corresponding triggering factors by normalizing $\mathrm{pH}$ and acute-phase proteins would shorten RBC rouleaux, which finally will disintegrate into single cells. In fact, we observed significant shortening of RBC stacks at hospital discharge compared with admission values (-Fig. 4). However, such trend was observed not in all patients, and some of them remained with long (3-4 cells) RBC stacks at discharge ( $\mathbf{- F i g}$. 4B). Second, it is unlikely that $\mathrm{RBC}$ glued in rouleaux can pass through the blood capillaries. Evidently, the aggregated RBC cannot deform synchronously to travel along the capillaries; they seemingly reach the venous bed via the arteriovenous anastomoses, not via capillaries. $^{25}$ The same considerations may be applied to echinocytes, which cannot reach the venous bed via the capillaries especially because they also form sludges. This pathway may explain why COVID-19 patients experience severe tissue hypoxia, although $\mathrm{SpO}_{2}$ in such patients is usually normal (>95\%). Our observation hinted that COVID-19 develops as pulmonary pathology only at the early stage of the disease, but during its progress, the vascular component of coronavirus infection begins to play an increasing damaging role.

Finally, at discharge, most biochemical inflammation indices normalized (-Table 2). The most common clinical symptom was remaining weakness, which is a natural manifestation of residual intoxication after viral infection. However, discharge electron microscopy images revealed that some patients $(22 \%)$ had remaining severe cell pa- thology indicative of vasculitis progression and multicellular RBC rouleaux (-Figs. 2 and 4 ). The cause of such discrepancy is unexplored but may be related to the differences in mitigating factors within the COVID-19 group. There is no doubt that such patients will benefit from frequent follow-up and further treatment, because they are probably still affected by so-called "long" COVID19. It is unclear how to triage such patients in clinics, since neither subjective assessment of their health nor the routine biochemical analyses can detect the latent and long-term development of disease. ${ }^{25}$ Future research should be focused on more uniformed and better matched groups identifying blood cell damage and impact of modern COVID-19 therapy. ${ }^{20}$

\section{Limitations}

The main shortcomings of this study were its retrospective cohort design and small sample size, which may limit the power to detect differences between arms. Obviously, these data are preliminary, and somewhat lack substance with no definite message. The COVID-19 group did not match ideally with the controls. Most importantly, there were solid differences in cell damage within the COVID-19 study arm, especially at hospital discharge. The short duration of follow-up might have limited our understanding of the delayed impact of COVID-19 on vascular-endothelial interplay, which is currently entirely unclear. Importantly, we also did not analyze the potential direct impact of COVID-19 on endothelium exclusively limiting our experiments to circulated cells. It will also be critical to prove whether the observed "holes" are indeed directly related to the virus invasion. Further experiments including in vitro assessment with ECs together with COVID-19 particles to reproduce similar damage will be mandatory to prove their direct influence. It will also be critical to define the impact of COVID-19 on the other major players such as neutrophils and platelets. Obviously, the index data are preliminary and descriptive with low incidence of parametric statistics due to the small sample size and substantial differences in clinical characteristics and electron microscopy indices.

\section{Conclusion}

COVID-19 is associated with persistent impairment of cellendothelium crosstalk. Not only the number of CECs was high, but also cell endothelium was massively damaged by multiple fenestrae in membranes with diameter comparable to the size of supercapsid in SARS-CoV-2 virus at hospital admission. COVID-19 also provoked formation of stacked aggregated erythrocytes capable of clogging microvascular bed and of diminishing oxygen supply. In some patients, such abnormalities persisted even at hospital discharge suggestive of a residual blood cell damage following the acute phase in some COVID-19 survivors. Such chronic sequelae can provoke long-term vascular complications following COVID-19, potentially requiring adequate protection including use of antithrombotics. 


\section{What is known about this topic?}

- Current COVID-19 pandemic reveals thrombotic, vascular, and endothelial dysfunctions at peak disease.

- However, the duration, degree of damage, and appropriate use of long-term antithrombotic strategies following acute phase are unclear.

- Most post-COVID data are yielded from random clinical observations or autopsy of postmortem samples, while precise cellular data in survivors are insufficient.

\section{What does this paper add?}

- Hospital admission number of circulating endothelial cells was significantly (40-100 times) higher in COVID19 patients.

- Cells were massively damaged by multiple fenestrae in membranes with diameter comparable to the size of supercapsid in SARS-CoV-2 virus.

- COVID-19 provokes formation of stacked aggregated erythrocytes capable of clogging microvascular bed and of diminishing oxygen supply.

- In some patients, such abnormalities persisted at hospital discharge revealing remaining intracellular penetration of COVID-19 where it may be replicated and returned to circulation.

\section{Funding}

This study was supported by the Russian Foundation for Basic Research (grant No. 19-015-00213) to Dr. Melkumyants.

\section{Conflict of Interest}

None declared.

\section{Acknowledgments}

We are indebted to Dr. Vyacheslav Golkov, the head of Nanopromimport Company of Science Park in Moscow State University and engineer Igor Bogdanov for excellent technical support. We are grateful to nurse Irina Varyushkina for collecting patient data.

\section{References}

1 Ackermann M, Verleden SE, Kuehnel M, et al. Pulmonary vascular endothelialitis, thrombosis, and angiogenesis in Covid-19. N Engl J Med 2020;383(02):120-128

2 Puelles VG, Lütgehetmann M, Lindenmeyer MT, et al. Multiorgan and renal tropism of SARS-CoV-2. N Engl J Med 2020;383(06): 590-592

3 Shafi AMA, Shaikh SA, Shirke MM, Iddawela S, Harky A. Cardiac manifestations in COVID-19 patients-A systematic review. J Card Surg 2020;35(08):1988-2008
4 Gupta A, Madhavan MV, Sehgal K, et al. Extrapulmonary manifestations of COVID-19. Nat Med 2020;26(07):1017-1032

5 Connors JM, Levy JH. Thromboinflammation and the hypercoagulability of COVID-19. J Thromb Haemost 2020;18(07):1559-1561

6 Batlle D, Soler MJ, Sparks MA, et al; COVID-19 and ACE2 in Cardiovascular, Lung, and Kidney Working Group. Acute kidney injury in COVID-19: emerging evidence of a distinct pathophysiology. J Am Soc Nephrol 2020;31(07):1380-1383

7 Zhang C, Shi L, Wang FS. Liver injury in COVID-19: management and challenges. Lancet Gastroenterol Hepatol 2020;5(05): 428-430

8 Hadi A, Werge M, Kristiansen KT, et al. Coronavirus disease-19 (COVID-19) associated with severe acute pancreatitis: case report on three family members. Pancreatology 2020;20(04):665-667

9 Aghagoli G, Marin BG, Katchur NJ, Chaves-Sell F, Asaad WF, Murphy SA. Neurological involvement in COVID-19 and potential mechanisms: a review. Neurocrit Care 2021;34(03):1062-1071

10 Sepehrinezhad A, Shahbazi A, Negah SS. COVID-19 virus may have neuroinvasive potential and cause neurological complications: a perspective review. J Neurovirol 2020;26(03):324-329

11 Varga Z, Flammer AJ, Steiger P, et al. Endothelial cell infection and endotheliitis in COVID-19. Lancet 2020;395(10234):1417-1418

12 Escher R, Breakey N, Lämmle B. Severe COVID-19 infection associated with endothelial activation. Thromb Res 2020;190:62

13 O'Sullivan JM, Gonagle DM, Ward SE, Preston RJS, O'Donnell JS. Endothelial cells orchestrate COVID-19 coagulopathy. Lancet Haematol 2020;7(08):e553-e555

14 Perrotta F, Matera MG, Cazzola M, Bianco A. Severe respiratory SARS-CoV2 infection: does ACE2 receptor matter? Respir Med 2020;168:105996

15 Loganathan S, Kuppusamy M, Wankhar W, et al. Angiotensinconverting enzyme 2 (ACE2): COVID 19 gate way to multiple organ failure syndromes. Respir Physiol Neurobiol 2021; 283:103548

16 Hoffmann M, Kleine-Weber H, Schroeder S, et al. SARS-CoV-2 cell entry depends on ACE2 and TMPRSS2 and is blocked by a clinically proven protease inhibitor. Cell 2020;181(02):271.e8-280.e8

17 Hamming I, Timens W, Bulthuis MLC, Lely AT, Navis G, van Goor H. Tissue distribution of ACE2 protein, the functional receptor for SARS coronavirus. A first step in understanding SARS pathogenesis. J Pathol 2004;203(02):631-637

18 Mancuso P, Gidaro A, Gregato G, et al. Circulating endothelial progenitors are increased in COVID-19 patients and correlate with SARS-CoV-2 RNA in severe cases. J Thromb Haemost 2020; 18(10):2744-2750

19 Moussa MD, Santonocito C, Fagnoul D, et al. Evaluation of endothelial damage in sepsis-related ARDS using circulating endothelial cells. Intensive Care Med 2015;41(02):231-238

20 Khider L, Gendron N, Goudot G, et al. Curative anticoagulation prevents endothelial lesion in COVID-19 patients. J Thromb Haemost 2020;18(09):2391-2399

21 Nizzoli ME, Merati G, Tenore A, et al. Circulating endothelial cells in COVID-19. Am J Hematol 2020;95(08):E187-E188

22 Guervilly C, Burtey S, Sabatier F, et al. Circulating endothelial cells as a marker of endothelial injury in severe COVID-19. J Infect Dis 2020;222(11):1789-1793

23 Lanuti P, Simeone P, Rotta G, et al. A standardized flow cytometry network study for the assessment of circulating endothelial cell physiological ranges. Sci Rep 2018;8(01):5823

24 Bouvier CA, Gaynor E, Cintron JR, Bernhardt B, Spaet TH. Circulating endothelium as an indication of vascular injury in vascular factors and thrombosis. Thromb Diath Haemorrh 1970;40:163

25 Al-Aly Z, Xie Y, Bowe B. High-dimensional characterization of post-acute sequelae of COVID-19. Nature 2021;594 (7862):259-264 